\title{
THE DEVELOPMENT AND STABILITY OF SOME NON-PLANAR BOUNDARY-LAYER FLOWS
}

\author{
NATHANIEL DAVID JEWELL
}

\author{
(Received 14 December 2009)
}

2000 Mathematics subject classification: primary 76E05; secondary 76D10, 76D17.

Keywords and phrases: fluid mechanics, hydrodynamic stability.

This thesis presents two problems in the field of fluid mechanics. Both problems concern the flow of a Newtonian viscous fluid in the laminar and early-transitional regimes. Geometrically, they also share the following features: a square corner; a wall boundary layer; and a semi-infinite physical domain.

Part 1 of this thesis, comprising Chapters 2-5, considers the laminar flow parallel to a streamwise corner. In Chapter 2 we present an in-depth study of the laminar flow internal to a square corner. The hydrodynamic stability of this flow is the subject of Chapter 3. For the special case of zero pressure gradient, our analysis suggests a critical Reynolds number of $\operatorname{Re}_{c} \approx 44000$ (based on streamwise distance from the leading edge), indicating that this flow is significantly less stable than the well-known Blasius boundary layer on a semi-infinite flat plate. In Chapter 4 we derive the laminar flow external to a square corner. Finally, in Chapter 5 we summarize our findings and offer some recommendations for future research on laminar and transitional corner flows.

Part 2, comprising Chapters 6-10, considers the sudden blockage of steady laminar flow within a circular pipe. Even though the blockage occurs almost instantaneously, the fluid takes an appreciable time to come to rest. Accordingly, Chapter 6 presents a detailed analysis of the laminar-decay process at an arbitrary location upstream of the blockage point. The hydrodynamic stability of this unsteady upstream flow is the subject of Chapters 7 and 8. Chapter 7 uses traditional linear eigenmode theory, originally developed for steady laminar flow, to estimate that the laminar flow is absolutely stable in the event that the pre-blockage Reynolds number does not exceed $\operatorname{Re}_{c} \approx 450$. The linear pseudomode analysis of Chapter 8 yields the substantially lower

Thesis submitted to the University of Adelaide, July 2009. Degree approved, December 2009. Supervisor: Associate Professor Jim Denier.

(C) 2010 Australian Mathematical Publishing Association Inc. 0004-9727/2010 \$16.00 
estimate $\operatorname{Re}_{c} \approx 115$, above which there exists the theoretical possibility of transient growth initiating a 'bypass' transition to turbulence. However, after accounting for the transient nature of the underlying flow itself, we hypothesize a significantly higher threshold $\operatorname{Re}_{c} \approx 1000$ for full breakdown of the laminar structure.

Chapter 9 rounds off the present work by extending the laminar-flow analysis of Chapter 6 to the immediate vicinity of the blockage point. We present a direct numerical simulation of the complete laminar-decay process within this end-region, highlighting the early-phase development of an unsteady corner boundary layer and the subsequent development of vortices in the interior of the pipe.

The thesis concludes in Chapter 10 by summarizing the findings from Part 2 and suggesting some fruitful directions for future research on unsteady pipe flows.

NATHANIEL DAVID JEWELL, School of Mathematical Sciences, The University of Adelaide, Adelaide, SA 5005, Australia

e-mail: nathaniel.jewell@adelaide.edu.au 